\title{
The role of tumor metabolism as a driver of prostate cancer progression and lethal disease: results from a nested case-control study
}

Rachel S. Kelly ${ }^{1,2,16^{*}}$, Jennifer A. Sinnott ${ }^{1,3}$, Jennifer R. Rider ${ }^{1,2}$, Ericka M. Ebot ${ }^{1}$, Travis Gerke ${ }^{1,4}$, Michaela Bowden ${ }^{5}$, Andreas Pettersson ${ }^{1,6}$, Massimo Loda ${ }^{5,7}$, Howard D. Sesso ${ }^{1,8}$, Philip W. Kantoff' ${ }^{9}$ Neil E. Martin ${ }^{10}$, Edward L. Giovannucci ${ }^{1,2,11}$, Svitlana Tyekucheva ${ }^{12}$, Matthew Vander Heiden ${ }^{13,14,15 \dagger}$ and Lorelei A. Mucci ${ }^{1,2+}$

\begin{abstract}
Background: Understanding the biologic mechanisms underlying the development of lethal prostate cancer is critical for improved therapeutic and prevention strategies. In this study we explored the role of tumor metabolism in prostate cancer progression using mRNA expression profiling of seven metabolic pathways; fatty acid metabolism, glycolysis/gluconeogenesis, oxidative phosphorylation, pentose phosphate, purine metabolism, pyrimidine metabolism and the tricarboxylic acid cycle.

Methods: The study included 404 men with archival formalin-fixed, paraffin-embedded prostate tumor tissue from the prospective Health Professionals Follow-up Study and Physicians' Health Study. Lethal cases $(n=113)$ were men who experienced a distant metastatic event or died of prostate cancer during follow-up. Non-lethal controls $(n=$ 291) survived at least 8 years post-diagnosis without metastases. Of 404 men, 202 additionally had matched normal tissue (140 non-lethal, 62 lethal). Analyses compared expression levels between tumor and normal tissue, by Gleason grade and by lethal status. Secondary analyses considered the association with biomarkers of cell proliferation, apoptosis and angiogenesis.

Results: Oxidative phosphorylation and pyrimidine metabolism were identified as the most dysregulated pathways in lethal tumors $(p<0.007)$, and within these pathways, a number of novel differentially expressed genes were identified including POLR2K and APT6V1A. The associations were tumor specific as there was no evidence any pathways were altered in the normal tissue of lethal compared to non-lethal cases.

Conclusions: The results suggest prostate cancer progression and lethal disease are associated with alterations in key metabolic signaling pathways. Pathways supporting proliferation appeared to be of particular importance in prostate tumor aggressiveness.
\end{abstract}

Keywords: Prostate cancer, mRNA expression profiling, Tumor metabolism, Metabolomic pathways, Tumorigenesis

\footnotetext{
* Correspondence: hprke@channing.harvard.edu

${ }^{\dagger}$ Equal contributors

'Department of Epidemiology, Harvard T.H. Chan School of Public Health,

Boston, MA, USA

${ }^{2}$ Channing Division of Network Medicine, Department of Medicine, Brigham

and Women's Hospital and Harvard Medical School, Boston, MA, USA

Full list of author information is available at the end of the article
}

(c) The Author(s). 2016 Open Access This article is distributed under the terms of the Creative Commons Attribution 4.0 International License (http://creativecommons.org/licenses/by/4.0/), which permits unrestricted use, distribution, and reproduction in any medium, provided you give appropriate credit to the original author(s) and the source, provide a link to the Creative Commons license, and indicate if changes were made. The Creative Commons Public Domain Dedication waiver (http://creativecommons.org/publicdomain/zero/1.0/) applies to the data made available in this article, unless otherwise stated. 


\section{Background}

It is well known that proliferating tumor cells have different metabolic requirements from normal, differentiated cells [1]. These metabolic needs are reflected, at least in part, by a shift in metabolic phenotype including the increased conversion of glucose to lactate even when oxygen is abundant, a phenomenon termed aerobic glycolysis or the Warburg effect [2]. Rapidly replicating cancer cells require the accumulation of sufficient biomass for cell growth, and increased glucose uptake is hypothesized to support these requirements [3, 4]. Consequently, tumors exhibit altered levels of many metabolites associated with cell growth as well as energetics, stress, and tissue-specific biochemistry [5, 6]. However, what is less well understood are the differences in metabolism between tumors by their degrees of severity.

Prostate cancer represents a particularly appropriate malignancy in which to explore this question. It is the second leading cause of cancer mortality in men in many Western countries, with more than 29,000 deaths in the USA in 2014 attributable to prostate cancer [7]. Yet, at the same time, most diagnoses will not prove fatal [5]. There is a wealth of evidence supporting an important role for dysregulated metabolism in prostate cancer. One of the most consistently cited risk factors is metabolic syndrome, a collection of patho-physiological entities including visceral obesity, insulin resistance, low HDL-cholesterol, high triglycerides, elevated C-reactive protein, and low adiponectin levels [8]. Crucially, the high concentrations of immune markers and the chronic inflammation associated with this syndrome are thought to enhance tumor growth [9]. suggesting that the metabolomic signatures of prostate tumors may also reflect their aggressive potential [5]. We recently reported that the pyrimidine biosynthesis pathway, which is responsible for production of nucleic acids needed for cell replication, is enriched in higher Gleason grade tumors [10]. Here we extend these analyses to explore other relevant metabolic pathways and to consider lethal disease as an endpoint.

The objective of this study was to investigate the metabolic pathways that underlie the development and progression of prostate cancer using an integrative molecular epidemiology approach. We characterized metabolic signatures at the messenger RNA (mRNA) level in prostate tumors and adjacent benign tissue among men with prostate cancer, and compared the expression profiles across Gleason grade and cancer outcomes. The analysis was focused on genes encoding enzymes involved in metabolic pathways to further characterize the role of dysregulated metabolism as a key driver in tumor aggressiveness and prostate cancer mortality. There is compelling experimental evidence to support this hypothesis; malignant cells are known to possess unique metabolic phenotypes that differ from the healthy tissues from which they originated [1-4], yet little data to date in a population-based study of prostate cancer patients. Specifically, we will focus on seven Kyoto Encyclopaedia of Genes and Genomes (KEGG) [11] defined pathways thought to be critical in tumor cellular metabolism, particularly within the prostate: fatty acid metabolism, glycolysis/gluconeogenesis, oxidative phosphorylation, pentose phosphate, purine metabolism, pyrimidine metabolism, and the tricarboxylic acid (TCA) cycle. By deconvoluting and quantifying the contribution of each of the pathways, and by isolating key driver genes within them, these findings will provide a uniquely comprehensive understanding of the metabolic processes underlying the development of lethal prostate cancer with considerable potential for direct translation to prostate cancer patients.

\section{Methods \\ Study population}

This study was nested among men with incident prostate cancer from the prospective US Health Professionals Follow-up Study (HPFS) or Physicians' Health Study (PHS). The HPFS [12] is a cohort study initiated in 1986 among 51,529 male health professionals aged 40 to 75 years. The PHS [13-15] was initiated as a randomized trial of aspirin and beta-carotene for the primary prevention of cardiovascular disease and cancer among 22,071 US male physicians aged 40 to 84 years.

Prostate cancer diagnosis is first reported on questionnaires and confirmed through medical record review. Clinical and pathological data including age at diagnosis, prostate-specific antigen (PSA) levels, and tumor stage are abstracted through medical record review. Postdiagnosis, men with prostate cancer are followed through questionnaires to collect information on their cancers' clinical course, including development of metastases. Cancer-specific and all-cause mortality is ascertained through mailings and telephone calls to participants, and periodic searches of the National Death Index. A committee of physicians assigns cause of death through medical record and death certificate review. Follow-up for mortality is available through 2011 and is $>98 \%$ complete.

This study includes 404 men with available archival formalin-fixed, paraffin-embedded (FFPE) prostate tumor tissue who were part of a whole genome expression profiling project of lethal prostate cancer: 150 men from PHS and 254 from HPFS. The study included lethal cases $(n=$ 113) defined as men with prostate cancer who experienced a distant metastatic event or died of prostate cancer during follow-up, and non-lethal prostate cancer cases $(n=291)$ who neither died of prostate cancer nor presented any evidence of metastases during follow-up and lived at least 8 years post-diagnosis. Mean follow- 
up was 13.2 years (range $0.1-27.4$ years). Of 404 men, 202 additionally had profiling for matched normal tissue (140 non-lethal cases, 62 lethal cases). Hematoxylin and eosin slides from all cases underwent standardized histopathologic review, including for Gleason grade and perineural invasion, by study pathologists [16].

\section{Tissue samples and mRNA profiling}

The validated mRNA profiling methodologies were described previously $[17,18]$. Briefly, RNA was extracted from two to three $0.6-\mathrm{mm}$ cores within tumor regions with high cell density ( $>80 \%$ cellularity) and normal tissue, then amplified using the WT-Ovation FFPE SystemV2 (Nugen, San Carlos, CA). Reverse transcription was used to create a complementary DNA (cDNA)/ mRNA hybrid, then the cDNA was amplified, fragmented, and labeled for hybridization to a GeneChip Human Exon 1.0 ST microarray (Affymetrics, Santa Clara, CA). The raw probe-level data was normalized through robust multichip averaging $[17,19]$ and probe annotation information obtained from the $\mathrm{R}$ package pd.hugene.1.0.st.v1 [20]. Probes not corresponding to genes were excluded, and where multiple probes corresponded to a single gene, the probe demonstrating the greatest variability was used. A total of 20,254 unique named genes were available. The genes comprising the specific metabolic pathways of interest were identified using the KEGG [11] and extracted from the whole transcriptome data.

The seven metabolic pathways are comprised of 444 unique genes. Of these, the expression profiles of 426 passed quality control criteria and were included in these analyses (Additional file 1: Table S1).

\section{Other tissue biomarkers}

Tumor biomarkers were available for a subset of participants, and these methods have been described previously. Cellular proliferation was characterized using Ki-67 staining [21], apoptosis using TUNEL staining [21], and tumor angiogenesis using microvessel density as defined by expression of endothelial cell marker CD34 [22].

\section{Statistical analysis}

Differences in baseline characteristics between the lethal and non-lethal cases were assessed using Student's $t$ test and the chi-squared test for continuous and categorical outcomes, respectively.

To explore the role of the metabolic pathways in disease progression, normalized expression levels were compared in tumor versus normal tissue, in lethal versus non-lethal cases, and in Gleason grade $\geq 8$ versus $2-7$ cases. Secondary analyses considered associations with markers of apoptosis, angiogenesis and cell proliferation, and the presence of perineural invasion. A variety of innovative statistical methods were integrated to capture and quantify both individual gene and pathway level effects.

Individual gene associations for each outcome were computed for the 426 genes using multivariable logistic regression models to estimate odds ratios and 95\% confidence intervals. Gene expression levels were modeled as continuous independent variables. Age at diagnosis, cohort (HPFS, PHS), year of diagnosis, and body mass index (BMI) at diagnosis were included as potential confounders. For within person tumor versus normal comparisons, conditional logistic regression was used.

Pathway level associations were explored using the Global test [23], a score test designed to detect effects across many genes in a pathway. The Global test was performed by comparing, for each KEGG pathway, a logistic regression model fitted with all the genes comprising that pathway and the potential confounders to a model including only the confounders. For models with secondary biomarkers divided into quartiles, a multicategory Global test was used. For the tumor-normal comparisons, matching was dropped for these pathway tests.

We also performed Gene Set Enrichment Analysis (GSEA) [24], a competitive test in which the differential expression of genes in the pathway is compared to differential expression of genes not involved in the pathway. GSEA determines the relative importance of the explored pathways and informs on the direction of effect. For tumor-normal comparisons, the genes were ranked according to their paired $t$ test statistic and the GSEA $p$ values were calculated using gene permutations; for the other analyses, standard GSEA was used with $p$ values calculated by permuting individuals.

Finally, a shrinkage and selection method, Least Absolute Shrinkage and Selection Operator (LASSO), was used to identify the genes contributing to pathway level associations and to determine the effect size. This approach fits a penalized regression model including all genes from each pathway as potential covariates and forces the gene expression coefficients not contributing to lethal outcome to zero so that they are removed from the model. The amount of shrinkage applied to the coefficients depends on a tuning parameter, which was chosen by using leave-one-out cross-validation to optimize the likelihood.

All analyses were conducted using the $\mathrm{R}$ software package.

\section{Results}

Table 1 presents the clinical features of the lethal and non-lethal cases. Lethal cases were more likely to be older and to have a higher Gleason grade, tumor stage, and PSA level at diagnosis. They were also more likely to have a higher BMI at both baseline and diagnosis. 
Table 1 Baseline and clinical characteristics of 404 participants with prostate cancer from the PHS and HPFS

\begin{tabular}{lll}
\hline Characteristic & Non-lethal $(n=291)$ & Lethal $(n=113)$ \\
\hline Cohort, N (\%) & & \\
PHS & $120(41.2 \%)$ & $30(26.5 \%)$ \\
HPFS & $171(58.8 \%)$ & $83(73.5 \%)$ \\
Age at diagnosis, mean (SD) & $64.9(6.2)$ & $67.5(6.7)$ \\
Clinical tumor stage, N (\%) & & \\
T1/T2 No/Nx M0/Mx & $271(94.1 \%)$ & $79(72.5 \%)$ \\
T3 N0/Nx M0/Mx & $16(5.6 \%)$ & $11(10.1 \%)$ \\
T4/N1/M1 & $1(0.3 \%)$ & $19(17.4 \%)$ \\
Gleason grade, N (\%) & & $1(0.9 \%)$ \\
2-6 & $56(19.2 \%)$ & $13(11.5 \%)$ \\
3+4 & $126(43.3 \%)$ & $35(31.0 \%)$ \\
$4+3$ & $67(23.0 \%)$ & $64(56.6 \%)$ \\
8-10 & $42(14.4 \%)$ & $25.6(3.2)$ \\
PSA at diagnosis, ng/ml, N (\%) & & $62(54.9)$ \\
0-3.9 & $29(10.7 \%)$ & $4(5.7 \%)$ \\
$4-10$ & $163(60.1 \%)$ & $35(50.0 \%)$ \\
10-19.9 & $54(19.9 \%)$ & $15(21.4 \%)$ \\
>20 & $25(9.2 \%)$ & $16(22.9 \%)$ \\
Tissue from RP, N (\%) & $283(97.3 \%)$ & $86(76.1 \%)$ \\
BMl at diagnosis, mean (SD) & $25.1(2.8)$ & $25.9(3.3)$ \\
BMl at baseline, mean (SD) & $24.6(2.5)$ & $140(48.1 \%)$ \\
Matched normal tissue available & & \\
\hline
\end{tabular}

${ }^{a}$ Clinical tumor stage was unknown for 3 (1\%) non-lethal cases and 4 (3.5\%) lethal cases

bPA was unknown for 20 (6.9\%) non-lethal cases and $43(38.1 \%)$ lethal cases

\section{Associations of metabolic pathways with tumorigenesis}

A total of 247 (58\%) genes in the metabolic pathways were differentially expressed in tumor vs. normal tissue $(p \leq 0.05)$ (Additional file 1: Table S2), and 118 (28\%) retained significance after Bonferroni correction for multiple testing at $p<1.2 \times 10^{-4}(0.05 / 426)$. The most significantly overexpressed genes in tumor tissue were
CANT1 (OR18.5, $\left.p=5.07 \times 10^{-13}\right)$, CMPK1 (OR 18.9, $\left.p=2.35 \times 10^{-12}\right)$, and GUCY1A3 (OR 8.0, $p=2.52 \times 10^{-12}$ ) annotated with the purine and pyrimidine pathways; FBP1 (OR 12.8, $p=2.75 \times 10^{-12}$ ) and GPI (OR 60.7, $p=$ $\left.2.19 \times 10^{-12}\right)$ annotated with the pentose phosphate and glycolysis pathways. The most significantly downregulated gene in tumor tissue was $A L D H 3 A 2$ (OR 0.03, $p=$ $3.23 \times 10^{-12}$ ) annotated with the KEGG glycolysis and fatty acid metabolism pathways. The differences in mRNA expression between tumor versus normal tissue were similar among the lethal and non-lethal cases (results not shown).

All seven pathways showed altered expression between tumor and adjacent normal prostate tissue according to the Global test $\left(p<1 \times 10^{-10}\right)$ (Table 2). Using GSEA testing, pyrimidine metabolism $(p=0.002)$, purine metabolism $(p=0.006)$, and oxidative phosphorylation $(p<$ $0.0001)$ were significant at a Bonferroni corrected threshold of $p<0.007$ (Table 3 ). All pathways were upregulated in the tumor versus normal tissue.

LASSO regression was then used to identify the genes contributing to the observed pathway level associations. Most of the significant associations for each pathway under the individual gene tests were also identified by the LASSO regression, and several additional genes were suggested to be of importance (Additional file 1: Table S2). These may represent genes that are important in a pathway context, when all other potentially interacting genes are taken into account.

\section{Associations of metabolic pathways with Gleason grade} We next compared tumor expression levels of the metabolic genes in Gleason 2-7 tumors vs. Gleason $\geq 8$ tumors (Additional file 1: Figure S1). A total of 101 (24\%) genes were associated with Gleason grade $(p \leq 0.05)$, of which six genes retained significance after Bonferroni correction. HPRT1 (purine metabolism pathway, OR 5.2, $p=1.93 \times 10^{-6}$ ), RRM2 (purine and pyrimidine metabolism pathways, $\mathrm{OR}=3.8, p=6.50 \times 10^{-6}$ ), PDE4D (purine

Table 2 Logistic Global test $p$ values for pathway level associations of metabolic pathways with tumorigenesis, Gleason grade, and lethal disease

\begin{tabular}{llll}
\hline Pathway $(n$ genes) & $\begin{array}{l}\text { Tumor tissue vs normal tissue } \\
(n=202)\end{array}$ & $\begin{array}{l}\text { Gleason grade }>8 \text { tumors vs Gleason grade } \\
2-7 \text { tumors }(n=404 ;>8=106 / 2-7=298)\end{array}$ & $\begin{array}{l}\text { Lethal tumors vs. non-lethal tumors } \\
(n=404 ; \mathrm{L}=113 / \mathrm{NL}=291)\end{array}$ \\
\hline Fatty acid metabolism $(n=39)$ & $<1.0 \times 10^{-10}$ & 0.03 & $1.4 \times 10^{-4}$ \\
Glycolysis/gluconeogenesis $(n=62)$ & $<1.0 \times 10^{-10}$ & $1.4 \times 10^{-5}$ & $<1.0 \times 10^{-10}$ \\
Pentose phosphate $(n=27)$ & $<1.0 \times 10^{-10}$ & $3.5 \times 10^{-3}$ & $8.9 \times 10^{-5}$ \\
Purine metabolism $(n=157)$ & $<1.0 \times 10^{-10}$ & $8.8 \times 10^{-6}$ & $1.2 \times 10^{-8}$ \\
Pyrimidine metabolism $(n=96)$ & $<1.0 \times 10^{-10}$ & $7.6 \times 10^{-5}$ & $1.9 \times 10^{-7}$ \\
Oxidative phosphorylation $(n=123)$ & $<1.0 \times 10^{-10}$ & $8.3 \times 10^{-5}$ & $3.5 \times 10^{-6}$ \\
TCA $(n=30)$ & $<1.0 \times 10^{-10}$ & 0.78 & 0.21 \\
\hline
\end{tabular}

All tests compare the null model including only age at diagnosis, cohort (PHS, HPFS), year of diagnosis, and BMI at diagnosis to the full model which also includes the genes in the listed pathway. Matching information is dropped for the tumor vs. normal comparison

$N L$ non-lethal, $L$ lethal 
Table $3 p$ value and direction of upregulation of the seven metabolic pathways for tumorigenesis, Gleason grade, and lethal prostate cancer, according to the Gene Set Enrichment Analysis

\begin{tabular}{|c|c|c|c|c|c|c|}
\hline \multirow[t]{2}{*}{ Pathway (n genes) } & \multicolumn{2}{|c|}{$\begin{array}{l}\text { Tumor tissue vs normal } \\
\text { tissue }(n=202)\end{array}$} & \multicolumn{2}{|c|}{$\begin{array}{l}\text { Gleason grade }>8 \text { tumors vs Gleason grade } \\
2-7 \text { tumors }(n=404 ;>8=106 / 2-7=298)\end{array}$} & \multicolumn{2}{|c|}{$\begin{array}{l}\text { Lethal tumors vs. non-lethal tumors } \\
(n=404 ; L=113 / \mathrm{NL}=291)\end{array}$} \\
\hline & $p$ value & $\begin{array}{l}\text { Expression in tumor } \\
\text { tissue }\end{array}$ & $p$ value & $\begin{array}{l}\text { Expression in high Gleason } \\
\text { grade }(>8) \text { tumors }\end{array}$ & $p$ value & $\begin{array}{l}\text { Expression in lethal } \\
\text { tumors }\end{array}$ \\
\hline $\begin{array}{l}\text { Fatty acid metabolism } \\
(n=39)\end{array}$ & 0.70 & $\uparrow$ & 0.37 & $\downarrow$ & 0.14 & $\downarrow$ \\
\hline $\begin{array}{l}\text { Glycolysis/gluconeogenesis } \\
(n=62)\end{array}$ & 0.18 & $\uparrow$ & 0.75 & $\uparrow$ & 0.44 & $\downarrow$ \\
\hline Pentose phosphate $(n=27)$ & 0.03 & $\uparrow$ & 0.21 & $\uparrow$ & 0.73 & $\uparrow$ \\
\hline Purine metabolism ( $n=157)$ & $6.0 \times 10^{-3}$ & $\uparrow$ & 0.03 & $\uparrow$ & 0.07 & $\uparrow$ \\
\hline $\begin{array}{l}\text { Pyrimidine metabolism } \\
(n=96)\end{array}$ & $2.0 \times 10^{-3}$ & $\uparrow$ & $3.0 \times 10^{-3}$ & $\uparrow$ & $6.0 \times 10^{-3}$ & $\uparrow$ \\
\hline $\begin{array}{l}\text { Oxidative phosphorylation } \\
(n=123)\end{array}$ & $<0.0001$ & $\uparrow$ & $4.0 \times 10^{-3}$ & $\uparrow$ & $6.0 \times 10^{-3}$ & $\uparrow$ \\
\hline TCA $(n=30)$ & 0.05 & $\uparrow$ & 0.64 & $\uparrow$ & 0.76 & $\uparrow$ \\
\hline
\end{tabular}

For the tumor vs. normal comparison matching is maintained and the GSEA-Preranked procedure is used with gene-based permutation $p$ values; for the other two comparisons, standard GSEA is used with sample-based permutation $p$ values

metabolism pathway, OR $\left.0.3, p=1.22 \times 10^{-5}\right)$, and NDUFC2 (oxidative phosphorylation pathway, $\mathrm{OR}=$ 3.6, $p=1.64 \times 10^{-5}$ ) ranked as the most significant (Additional file 1: Table S3). Results were similar when Gleason 7 cancers $(3+4$ and $4+3)$ were excluded and Gleason 2-6 vs. Gleason 8-10 tumors were compared (results not shown).

At a pathway level, purine metabolism $\left(p=8.8 \times 10^{-6}\right)$, glycolysis/gluconeogenesis $\left(p=1.37 \times 10^{-5}\right)$, pyrimidine metabolism $\left(p=7.56 \times 10^{-5}\right)$, and oxidative phosphorylation $\left(p=8.3 \times 10^{-5}\right)$ were significant under the Global test (Table 2). Pyrimidine metabolism ( $p=$ $0.003)$ and oxidative phosphorylation $(p=0.004)$ were also significant under GSEA (Table 3). Most of the significant genes identified using logistic regression also ranked under the LASSO model, but novel genes were also identified.

\section{Associations of metabolic pathways with lethal status}

Tumor expression of 124 genes was associated with lethal vs. non-lethal outcome $(p \leq 0.05)$. The associated odds ratios and $p$ values are described in Fig. 1 . Threequarters $(n=98,76 \%)$ of these genes were more highly expressed in the lethal cases (Additional file 1: Table S4). Twenty-six genes retained significance after Bonferroni correction. POLR $2 K$, part of the purine and pyrimidine metabolism pathways, ranked as the top differentially expressed gene (OR 8.6, $p=5.84 \times 10^{-9}$ ). RRM2 (OR 6.8 $\left.p=7.72 \times 10^{-9}\right)$, POLE2 (OR 13.2, $p=7.36 \times 10^{-7}$ ) (both associated with purine and pyrimidine metabolism), and ATP6V1A (OR 6.9, $p=7.81 \times 10^{-8}$ ) (oxidative phosphorylation) were also expressed to higher levels in lethal tumors, while $A L D H 2$ (OR 0.3, $p=4.65 \times 10^{-8}$ (associated with fatty acid metabolism and glycolysis), PDE4D (OR
$0.2, p=7.14 \times 10^{-8}$, purine metabolism), and $A L D H 1 A 3$ (OR $0.4, p=1.89 \times 10^{-6}$, glycolysis) ranked as the most significantly downregulated genes. The associations with lethal disease were attenuated but still significant when adjusting for Gleason grade (Additional file 1: Figure S2).

All but the TCA cycle pathway were significantly associated with lethal disease by the Global test at the Bonferroni corrected threshold (Table 2). Most significant associations retained significance or borderline significance when additionally adjusting for Gleason grade (Additional file 1: Table S5).

To determine whether the metabolic pathway gene expression changes may be influenced by gene expression changes in the surrounding tissue, expression levels were compared in the morphologically normal prostate tissue from lethal cases $(n=62)$ with the normal tissue from non-lethal $(n=140)$ cases. None of the pathways were identified as significant according to the Global test $(p>$ 0.05 for all pathways), and thus the relationship between lethal disease and altered metabolic gene expression appears to be a tumor-specific effect.

Intriguingly, the strongest associations were apparent in the overweight/obese men (Additional file 1: Table S5). There was some evidence that the associations were restricted to low-stage (T1/T2 N0/Mx) tumors, although the stage-stratified analyses were limited by small numbers. Again the pathways of pyrimidine metabolism $(p=0.006)$ and oxidative phosphorylation $(p=0.006)$ were significant under GSEA analysis (Table 3), with both pathways upregulated in the lethal compared to non-lethal tumors. The drivers of these pathway associations were explored using LASSO regression (Additional file 1: Table S4), and the findings suggested a role for both the top hits from 


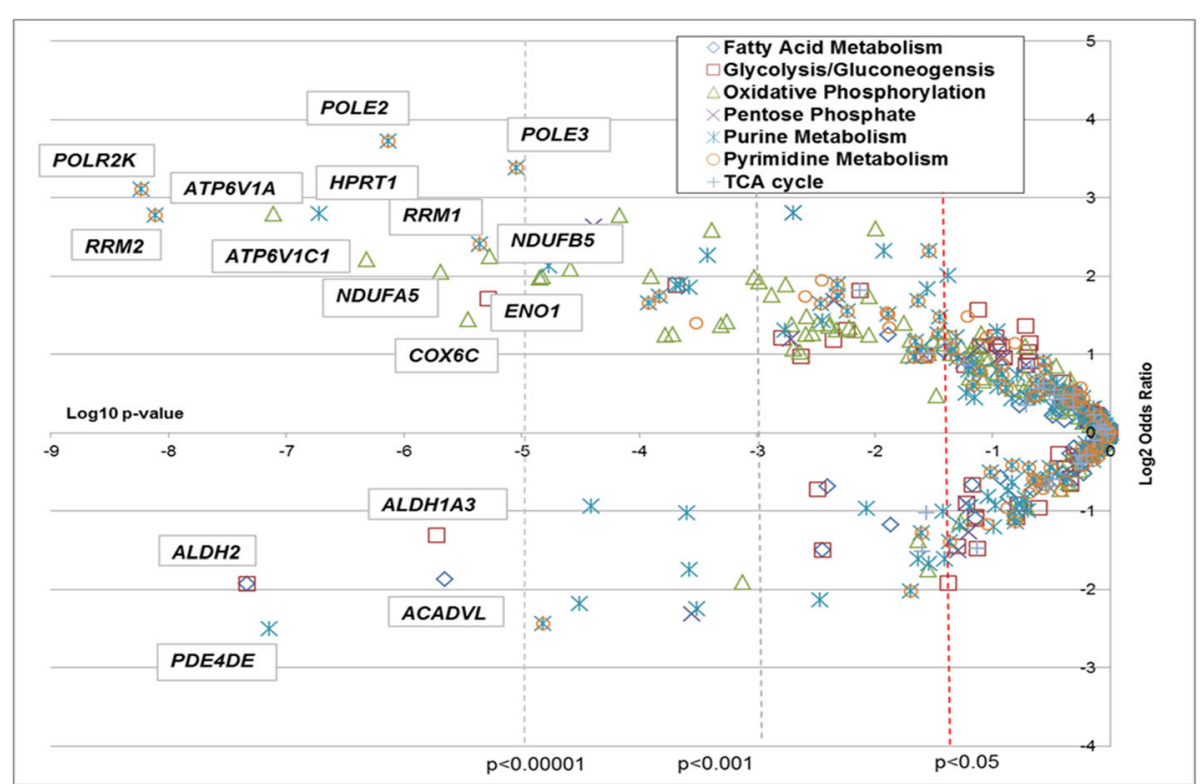

Fig. 1 Strength and significance of individual gene associations with lethal prostate cancer among seven metabolic pathways. Log odds ratios computed using a logistic regression model adjusting for age at diagnosis, cohort (HPFS, PHS), year of diagnosis, and BMI at diagnosis

the logistic regression model as well as a number of additional genes that may be acting jointly on risk.

\section{Associations with additional secondary outcomes}

We explored the association between expression of the metabolic pathways with biomarkers of cell proliferation, apoptosis, angiogenesis, and perineural invasion. The strongest associations of the pathways were with cell proliferation $(p<0.007)$ (Table 4$)$. Conversely, there was no Bonferroni corrected significant association with apoptosis nor angiogenesis. Extent of perineural invasion was significantly associated with fatty acid metabolism $\left(p=1.1 \times 10^{-6}\right)$, glycolysis/gluconeogenesis $\left(p=1.2 \times 10^{-4}\right)$, purine metabolism $\left(p=1.9 \times 10^{-5}\right)$, and pyrimidine metabolism $\left(p=3.5 \times 10^{-4}\right)$.

\section{Discussion}

Cancer cells share a metabolic requirement to support inappropriate cell proliferation and maintain growth [1]; however, the ways in which these requirements are fulfilled differ across tumor types. In particular, the healthy prostate is known to exhibit a unique metabolism, enabling the production of the components of prostatic fluid: PSA, spermine, myso-inositol, and citrate. This is disrupted in neoplastic cells due to the loss of ability to accumulate zinc and subsequently to accumulate citrate. Therefore prostate tumors display unique metabolomic alterations $[25,26]$. These metabolic alterations may vary according to tumor aggressiveness; however, the majority of studies to date exploring this question have focused on cell lines or model organism. None have focused on prostate cancer within a patient cohort $[2,27]$. The

Table 4 Multicategory Global test $p$ value for pathway level associations of tumor expression of the metabolic pathways with histologic and molecular features of prostate cancer

\begin{tabular}{|c|c|c|c|c|}
\hline Pathway (n genes) & $\begin{array}{l}\text { Ki-67 quartiles } \\
(n=314 \text { men) }\end{array}$ & $\begin{array}{l}\text { Apoptosis quartiles } \\
\text { ( } n=255 \text { men) }\end{array}$ & $\begin{array}{l}\text { Microvessel density quartiles }{ }^{a} \\
(n=174 \text { men })\end{array}$ & $\begin{array}{l}\text { Perineural invasion }{ }^{a} \\
(n=132 \text { men) }\end{array}$ \\
\hline Fatty acid metabolism $(n=39)$ & $1.8 \times 10^{-4}$ & 0.20 & 0.01 & $1.1 \times 10^{-6}$ \\
\hline Glycolysis/gluconeogenesis ( $n=62$ ) & $9.4 \times 10^{-9}$ & 0.19 & 0.06 & $1.2 \times 10^{-4}$ \\
\hline Pentose phosphate $(n=27)$ & $4.0 \times 10^{-6}$ & 0.20 & 0.14 & 0.04 \\
\hline Purine metabolism ( $n=157)$ & $4.1 \times 10^{-6}$ & 0.42 & 0.09 & $1.9 \times 10^{-5}$ \\
\hline Pyrimidine metabolism $(n=96)$ & $2.8 \times 10^{-6}$ & 0.64 & 0.17 & $3.5 \times 10^{-4}$ \\
\hline Oxidative phosphorylation $(n=123)$ & $3.1 \times 10^{-8}$ & 0.09 & $2.0 \times 10^{-3}$ & 0.02 \\
\hline TCA $(n=30)$ & $1.1 \times 10^{-7}$ & 0.30 & 0.04 & 0.37 \\
\hline
\end{tabular}

All tests compare the null model including only age at diagnosis, cohort (PHS, HPFS) if data was available for both, year of diagnosis, and BMI at diagnosis to the full model which also includes the genes in the listed pathway

${ }^{a}$ Data was only available for HPFS so cohort was excluded from these models 
results of this novel study provide human-based evidence that key metabolic pathways are relevant to prostate cancer lethality. Therefore, the findings have the potential for forward translation into the identification of therapeutic targets and the development of biomarkers that better define tumors by their degree of aggressiveness.

Consistent with previous literature [3], in this study, we demonstrate alterations in key metabolic genes and pathways within the prostate during tumorigenesis. Crucially, we additionally offer evidence that these changes differ in cancer progression by histologic grade and by lethal outcome. Changes in prostate tumor gene expression were dominated by increased expression in the lethal or high-grade cancers, which may suggest that this subset of aggressive prostate tumors have increased metabolic activity. This is supported by studies in other tumors reporting increased nutrient uptake by FDGPET is associated with more aggressive cancer [28].

Among the top differentially expressed genes, many were common between the tumorigenesis, lethal and Gleason analyses, while others appeared to be specific to disease aggressiveness. A number of the top genes identified in this study have previously been implicated in prostate cancer development and progression, Gleason grade, metastases and biochemical recurrence; including CANT1 [29], FBP1 [30], RRM2 [31], POLE2 [32], PDE4D [33], and ALDH1A3 [34]. This supports the validity of our findings and strengthens the evidence for the role of these genes by demonstrating their differential expression levels in a human study.

Additionally, we report a number of novel genes with biological plausibility; this is the first study to report on the association between GPI and ALDH3A2 with prostate cancer in a human population. Mammalian GPI has been demonstrated to function as a tumor-secreted cytokine and angiogenic factor, while cells with high ALDH activity have been shown to display metastasis-initiating behavior [35]. A further ALDH isoform, ALDH2, has previously been associated with the progression of benign prostatic hyperplasia [36], but this study represents the first to specifically link it with lethal prostate cancer. Similarly, we report novel associations between Gleason grade with HPRT1 and NDUFC2. NADH dehydrogenase has been repeatedly implicated in prostate cancer risk [37], and both these genes play vital roles in the generation of the purine nucleotides necessary to support proliferation.

On a pathway level, the predominance of gene expression changes associated with glycolysis and oxidative phosphorylation pathways provides support for a shift in metabolism toward increased aerobic glycolysis in tumors with lethal potential [3]. Changes in gene expression in the other investigated pathways may help dividing cells fulfill proliferation requirements that extend beyond ATP production [2]. The increased expression of genes encoding enzymes in the purine and pyrimidine metabolism pathways in lethal relative to non-lethal tumors may support increased DNA replication and cell proliferation in these tumors, while changes in the pentose phosphate pathway and fatty acid metabolism genes may reflect the increased need of lethal tumors for nucleotides, amino acids, and lipids $[4,27]$. This is further supported by the association of these pathways with biomarkers of cell proliferation and is in agreement with the enrichment of genes of pyrimidine metabolism in high Gleason grade tumors [10]. Interestingly, there was no difference in metabolic enzyme expression of the seven KEGG pathways observed in the adjacent normal tissue from patients who developed lethal prostate cancer compared to those who did not develop lethal disease, supporting a tumorspecific effect.

There are potential limitations to this study. A significantly larger proportion of the non-lethal cases had biopsy tissue available from transurethral resection of the prostate as opposed to radical prostatectomy (RP), and our recent study suggests that the expression levels of some genes differs as a function of zone of origin [10]. However, sensitivity analyses restricting to the RP cases produced comparable results. A hypothesis-led approach was taken for the selection of metabolic pathways, although it is possible that other metabolic pathways not considered in these analyses may be exerting an effect. In this study, we were unable to measure metabolite concentrations and inferred the metabolic state based on expression of metabolic pathways. However, the regulatory mechanisms determining the relationship between gene expression and metabolite levels are complex and not yet fully understood [38]. Transcript levels and metabolite abundances do not overlap directly with the underlying biochemical pathways [39]. The correlations between transcripts and metabolites may be influenced by time lags, reaction kinetics, network effects, feedback reactions, or noise. Furthermore, post-translational modification can have substantial impacts on metabolite levels [38, 39].

Nevertheless, transcriptional regulation is known to play an important role in the control of metabolism [40], and thus can be considered a good proxy with which to explore our pathways of interest. This study represents one of the largest of its kind to date and, to the best of our knowledge, is the first to use in silico analysis of gene expression profiling to discover new hypotheses that may underlie lethal prostate cancer link. We focus on important but as yet underexplored hypotheses, based on strong biological rational, to provide an increased mechanistic understanding of lethal prostate 
cancer. The nesting within two population-based studies with a rich variety of epidemiological, anthropometric, and lifestyle data allows us to control for possible confounding factors and evaluate effect modifiers. Furthermore, the methodologies utilized in this study have been previously validated within our included cohorts. The availability of matched normal tissue allowed us to consider the potential role of the tumor microenvironment on our findings, and the possibility of a field effect, representing a further strength of this study.

\section{Conclusions}

In conclusion, prostate tumorigenesis is associated with dysregulation of key metabolic signaling pathways. Within tumor tissue, we offer evidence of specific alterations that differ by Gleason grade and lethal outcome and identify novel genes that may play an important role. Our findings add further support to the hypotheses that metabolic alterations in aggressive tumors may extend beyond an increase in aerobic glycolysis and encompass changes in gene expression for enzymes involved in other pathways supporting proliferation $[2,4,41]$. A thorough understanding of the metabolic phenotypes of aggressive tumors may help us to better define the mechanisms underlying lethal prostate cancer and illuminate novel therapeutic targets, to improve outcomes for those men at greatest risk of prostate cancer death $[2,4,42]$.

\section{Additional file}

Additional file 1: Table S1. The number of available genes from the seven selected KEGG-defined metabolic pathways and the number of common genes between the pathways, after the exclusion of genes that failed quality control. Table S2 Genes associated with tumorigenesis in 202 prostate cancer cases with tumor and normal tissue available according to a conditional logistic regression model and a Least Absolute Shrinkage and Selection Operator (LASSO) regression model. Table S3 Genes associated with Gleason grade (Grade 2-7 versus grade 8) in 404 prostate cancer cases according to a logistic regression model and a Least Absolute Shrinkage and Selection Operator (LASSO) regression model. Table S4 Genes associated with lethal disease in 404 prostate cancer cases (291 non-lethal and 113 lethal) cases according to a logistic regression model and a Least Absolute Shrinkage and Selection Operator (LASSO) regression model. Table S5 Global test $p$ value for pathway level associations with lethal prostate cancer exploring potential effect modifiers. Figure S1 Strength (log odds ratios) and significance (log $p$ values) of individual gene associations with Gleason grade $\geq 8(n=106)$ versus Gleason grade $2-7 \quad(n=298)$ tumors across the seven pathways. Figure $\mathbf{S} 2$ Strength (log odds ratios) and significance (log $p$ values) of individual gene associations with lethal prostate cancer among the seven metabolic pathways after additional adjustment for Gleason grade. (DOCX $337 \mathrm{~kb}$ )

\section{Abbreviations}

ATP: Adenosine triphosphate; BMI: TUNEL staining body mass index; FDGPET: Fludeoxyglucose - positron emission tomography; FFPE: Formalin-fixed, paraffin-embedded; GSEA: Gene Set Enrichment Analysis; HPFS: Health Professionals Follow-up Study; IRB: Institutional review boards; KEGG: Kyoto Encyclopaedia of Genes and Genomes; LASSO: Least Absolute Shrinkage and
Selection Operator; PHS: Physicians' Health Study; PSA: Prostate-specific antigen; RP: Radical prostatectomy; TCA: Tricarboxylic acid

\section{Acknowledgements}

We are grateful to the participants and staff of the Physicians' Health Study and Health Professionals Follow-Up Study for their valuable contributions. We thank Elizabeth Nuttall and Michael Pitt for valuable assistance in the whole genome gene expression profiling data. We would like to acknowledge the key contributions of Alex Forrest Hay at Affymetrix and Dr. Edward Fox (in memorium) at the Dana-Farber Cancer Institute Microarray Core. In addition, we would like to thank the following state cancer registries for their cooperation and assistance: $A L, A Z, A R, C A, C O, C T, D E, F L, G A, I D, I L$, IN, IA, KY, LA, ME, MD, MA, MI, NE, NH, NJ, NY, NC, ND, OH, OK, OR, PA, RI, SC, TN, TX, VA, WA, WY

\section{Funding}

This work was supported by the DF/HCC Specialized Programs in Research Excellence (SPORE) in Prostate Cancer funded by the NCI/NIH (Grant number: 2P50CA090381-11A1 Pl: Philip Kantoff, MD), P01 CA055075, CA133891,

CA141298, CA136578 and UM1 CA167552. The Physicians' Health Study was additionally supported by CA097193, CA34944, CA40360, HL26490, and HL34595 from the National Institutes of Health (Bethesda, MD USA). JAS was supported by the National Institutes of Health (NIH) grant T32 CA09001 and the A.David Mazzone Career Development Award. LAM, JRR, and NEM are Young Investigators of the Prostate Cancer Foundation. The funder had no role in the design and conduct of the study; collection, management, analysis, and interpretation of the data; preparation, review, or approval of the manuscript; or in the decision to submit the manuscript for publication.

\section{Availability of data and materials}

The dataset supporting the conclusions of this article is currently being prepared for submission to the Gene Expression Omnibus (GEO), pending the publication of additional manuscripts utilizing this data.

\section{Authors' contributions}

All authors were involved in the conception and design of the project or the acquisition of the included data, and all authors critically revised the manuscript for important intellectual content. ML, HDS, PWK, NEM, ELG, $\mathrm{MVH}$, and LAM were involved in obtaining funding to support the project. JRR, EE, MB, AP, and ST provided technical, laboratory or other support. LAM and MVH were the primary supervisors and mentors of RSK. RSK conducted the data analysis with support from JAS and TG. RSK had full access to all the data in the study and takes responsibility for the integrity of the data and the accuracy of the data analysis. All authors read and approved the final manuscript.

\section{Competing interests}

The authors declare that they have no competing interests.

\section{Consent for publication}

Not applicable

\section{Ethics approval and consent to participate}

The study was approved by the institutional review boards (IRB) at the Harvard T.H. Chan School of Public Health and Partners Healthcare. All tumor samples were collected using IRB-approved processes.

\section{Author details}

${ }^{1}$ Department of Epidemiology, Harvard T.H. Chan School of Public Health, Boston, MA, USA. ${ }^{2}$ Channing Division of Network Medicine, Department of Medicine, Brigham and Women's Hospital and Harvard Medical School, Boston, MA, USA. ${ }^{3}$ Department of Biostatistics, Harvard T.H. Chan School of Public Health, Boston, MA, USA. ${ }^{4}$ Department of Epidemiology, College of Medicine and College of Public Health and Health Professions, University of Florida, Gainesville, FL, USA. ${ }^{5}$ Center for Molecular Oncologic Pathology, Dana-Farber Cancer Institute, Boston, MA, USA. ${ }^{6}$ Clinical Epidemiology Unit, Department of Medicine Solna, Karolinska Institutet, Stockholm, Sweden. ${ }^{7}$ Department of Pathology, Brigham and Women's Hospital and Harvard Medical School, Boston, MA, USA. ${ }^{8}$ Division of Preventive Medicine, Brigham and Women's Hospital and Harvard Medical School, Boston, MA, USA.

${ }^{9}$ Department of Medical Oncology, Dana-Farber Cancer Institute, Harvard 
Medical School, Boston, MA, USA. ${ }^{10}$ Department of Radiation Oncology, Brigham and Women's Hospital/Dana-Farber Cancer Institute, Harvard Medical School, Boston, MA, USA. ${ }^{11}$ Department of Nutrition, Harvard T.H. Chan School of Public Health, Boston, MA, USA. ${ }^{12}$ Department of Biostatistics and Computational Biology, Dana-Farber Cancer Institute, Boston, MA, USA.

${ }^{13}$ Koch Institute for Integrative Cancer Research at Massachusetts Institute of Technology, Cambridge, MA 02139, USA. ${ }^{14}$ Dana-Farber Cancer Institute, Boston, MA, USA. ${ }^{15}$ Broad Institute of Harvard and Massachusetts Institute of Technology, Cambridge, MA 02139, USA. ${ }^{16}$ Channing Division of Network Medicine, 181 Longwood Avenue, Boston, MA 02115, USA.

\section{Received: 4 May 2016 Accepted: 9 November 2016} Published online: 07 December 2016

\section{References}

1. Warburg O, Wind F, Negelein E. The Metabolism of Tumors in the Body. J Gen Physiol. 1927:8:519-30.

2. Vander Heiden MG, Cantley LC, Thompson CB. Understanding the Warburg Effect: The Metabolic Requirements of Cell Proliferation. Science (New York, NY). 2009;324:1029-33.

3. Hu J, Locasale JW, Bielas JH, O'Sullivan J, Sheahan K, Cantley LC, Vander Heiden MG, Vitkup D. Heterogeneity of tumor-induced gene expression changes in the human metabolic network. Nat Biotechnol. 2013;31:522-9.

4. Vander Heiden MG. Targeting cancer metabolism: a therapeutic window opens. Nat Rev Drug Discov. 2011;10:671-84.

5. McDunn JE, Li Z, Adam K-P, Neri BP, Wolfert RL, Milburn MV, Lotan Y, Wheeler TM. Metabolomic signatures of aggressive prostate cancer. Prostate. 2013;73:1547-60.

6. Spratlin JL, Serkova NJ, Eckhardt SG. Clinical Applications of Metabolomics in Oncology: A Review. Clin Cancer Res. 2009;15:431-40.

7. Detailed Guide: Prostate Cancer [http://www.cancer.org/cancer/prostatecancer/ detailedguide/prostate-cancer-key-statistics] Accessed May 2016.

8. McGrowder DA, Jackson LA, Crawford TV. Prostate cancer and metabolic syndrome: is there a link? Asian Pac J Cancer Prev. 2012;13:1-13.

9. Hsing AW, Sakoda LC, Chua SC. Obesity, metabolic syndrome, and prostate cancer. Am J Clin Nutr. 2007:86:843S-57.

10. Penney KL, Sinnott JA, Fall K, Pawitan $Y$, Hoshida $Y$, Kraft P, Stark JR, Fiorentino M, Perner S, Finn S, et al. mRNA Expression Signature of Gleason Grade Predicts Lethal Prostate Cancer. J Clin Oncol. 2011;29:2391-6.

11. Kanehisa M, Goto S. KEGG: kyoto encyclopedia of genes and genomes. Nucleic Acids Res. 2000;28:27-30

12. Rider JR, Fiorentino M, Kelly R, Gerke T, Jordahl K, Sinnott JA, Giovannucci EL, Loda M, Mucci LA, Finn S, Transdisciplinary Prostate Cancer Partnership (ToPCaP). Tumor expression of adiponectin receptor 2 and lethal prostate cancer. Carcinogenesis. 2015;36(6):639-47.

13. Christen WG, Gaziano JM, Hennekens CH. Design of Physicians' Health Study $\|$-a randomized trial of beta-carotene, vitamins $E$ and $C$, and multivitamins, in prevention of cancer, cardiovascular disease, and eye disease, and review of results of completed trials. Ann Epidemiol. 2000;10:125-34.

14. Steering Committee of the Physicians' Health Study Research Group. Final Report on the Aspirin Component of the Ongoing Physicians' Health Study. N Engl J Med. 1989;321:129-35.

15. Hennekens $\mathrm{CH}$, Buring JE, Manson JE, Stampfer M, Rosner B, Cook NR, Belanger C, LaMotte F, Gaziano JM, Ridker PM, et al. Lack of Effect of Long Term Supplementation with Beta Carotene on the Incidence of Malignant Neoplasms and Cardiovascular Disease. N Engl J Med. 1996;334:1145-9.

16. Stark JR, Perner S, Stampfer MJ, Sinnott JA, Finn S, Eisenstein AS, Ma J, Fiorentino M, Kurth T, Loda M, et al. Gleason score and lethal prostate cancer: does $3+4=4+3$ ? J Clin Oncol. 2009:27:3459-64.

17. Penney KL, Sinnott JA, Tyekucheva S, Gerke T, Shui IM, Kraft P, Sesso HD, Freedman ML, Loda M, Mucci LA, Stampfer MJ. Association of Prostate Cancer Risk Variants with Gene Expression in Normal and Tumor Tissue. Cancer Epidemiol Biomark Prev. 2015;24:255-60.

18. Tyekucheva S, Martin NE, Stack EC, Wei W, Vathipadiekal V, Waldron L, Fiorentino M, Lis RT, Stampfer MJ, Loda M, et al. Comparing Platforms for Messenger RNA Expression Profiling of Archival Formalin-Fixed, ParaffinEmbedded Tissues. J Mol Diagn. 2015;17(4):374-381.

19. Irizarry RA, Bolstad BM, Collin F, Cope LM, Hobbs B, Speed TP. Summaries of Affymetrix GeneChip probe level data. Nucleic Acids Res. 2003;31:e15.

20. Carvalho B. pd.hugene.1.0.st.v1: Platform Design Info for Affymetric HuGene1_0-st-v1. version 3.8.0 edition. pp. R package; 2014:R package.
21. Zu K, Martin NE, Fiorentino M, Flavin R, Lis RT, Sinnott JA, Finn S, Penney KL, Ma J, Fazli L, et al. Protein Expression of PTEN, Insulin-Like Growth Factor I Receptor (IGF-IR), and Lethal Prostate Cancer: A Prospective Study. Cancer Epidemiol Biomarkers Prev. 2013;22:1984-93.

22. Mucci LA, Powolny A, Giovannucci E, Liao Z, Kenfield SA, Shen R, Stampfer MJ, Clinton SK. Prospective study of prostate tumor angiogenesis and cancer-specific mortality in the health professionals follow-up study. J Clin Oncol. 2009;27:5627-33.

23. Goeman JJ, Van De Geer SA, Van Houwelingen HC. Testing against a high dimensional alternative. J R Stat Soc Ser B (Stat Methodol). 2006;68:477-93.

24. Subramanian A, Tamayo P, Mootha VK, Mukherjee S, Ebert BL, Gillette MA, Paulovich A, Pomeroy SL, Golub TR, Lander ES, Mesirov JP. Gene set enrichment analysis: A knowledge-based approach for interpreting genome-wide expression profiles. Proc Natl Acad Sci. 2005;102:15545-50.

25. Trock BJ. Application of Metabolomics to Prostate Cancer. Urol Oncol. 2011; 29:572-81.

26. Costello LC, Franklin RB. Concepts of citrate production and secretion by prostate. 1. Metabolic relationships. Prostate. 1991;18:25-46.

27. Lunt SY, Vander Heiden MG. Aerobic Glycolysis: Meeting the Metabolic Requirements of Cell Proliferation. Annu Rev Cell Dev Biol. 2011;27:441-64.

28. Kubota K. From tumor biology to clinical Pet: a review of positron emission tomography (PET) in oncology. Ann Nucl Med. 2001;15:471-86.

29. Gerhardt J, Steinbrech C, Büchi O, Behnke S, Bohnert A, Fritzsche F, Liewen $\mathrm{H}$, Stenner F, Wild P, Hermanns T, et al. The Androgen-Regulated CalciumActivated Nucleotidase 1 (CANT1) Is Commonly Overexpressed in Prostate Cancer and Is Tumor-Biologically Relevant in Vitro. Am J Pathol. 2011;178: 1847-60.

30. Weber A, Kristiansen I, Johannsen M, Oelrich B, Scholmann K, Gunia S, May M, Meyer H-A, Behnke S, Moch H, Kristiansen G. The FUSE binding proteins FBP1 and FBP3 are potential c-myc regulators in renal, but not in prostate and bladder cancer. BMC Cancer. 2008:8:1-11.

31. Huang Y, Liu X, Wang Y-H, Yeh S-D, Chen C-L, Nelson RA, Chu P, Wilson T, Yen $Y$. The prognostic value of ribonucleotide reductase small subunit M2 in predicting recurrence for prostate cancers. Urol Oncol. 2014;32:51.e59-51. e19.

32. Malhotra S, Lapointe J, Salari K, Higgins JP, Ferrari M, Montgomery K, van de Rijn M, Brooks JD, Pollack JR. A Tri-Marker Proliferation Index Predicts Biochemical Recurrence after Surgery for Prostate Cancer. PLoS ONE. 2011;6:e20293.

33. Rahrmann EP, Collier LS, Knutson TP, Doyal ME, Kuslak SL, Green LE, Malinowski RL, Roethe L, Akagi K, Waknitz M, et al. Identification of PDE4D as a Proliferation Promoting Factor in Prostate Cancer Using a Sleeping Beauty Transposon-Based Somatic Mutagenesis Screen. Cancer Res. 2009;69:4388-97.

34. Le Magnen C, Bubendorf L, Rentsch CA, Mengus C, Gsponer J, Zellweger T, Rieken M, Thalmann GN, Cecchini MG, Germann M, et al. Characterization and Clinical Relevance of ALDHbright Populations in Prostate Cancer. Clin Cancer Res. 2013;19:5361-71.

35. van den Hoogen $C$, van der Horst G, Cheung H, Buijs JT, Lippitt JM, GuzmánRamírez N, Hamdy FC, Eaton CL, Thalmann GN, Cecchini MG, et al. High Aldehyde Dehydrogenase Activity Identifies Tumor-Initiating and MetastasisInitiating Cells in Human Prostate Cancer. Cancer Res. 2010;70:5163-73.

36. Seok H, Yoo KH, Kim YO, Chung J-H. Association of a Missense ALDH2 Single Nucleotide Polymorphism (Glu504Lys) With Benign Prostate Hyperplasia in a Korean Population. Int Neurourol J. 2013;17:168-73.

37. Wang L, McDonnell SK, Hebbring SJ, Cunningham JM, Sauver JS, Cerhan JR, Isaya G, Schaid DJ, Thibodeau SN. Polymorphisms in mitochondrial genes and prostate cancer risk. Cancer Epidemiol Biomark Prev. 2008;17:3558-66.

38. Zelezniak A, Sheridan S, Patil KR. Contribution of Network Connectivity in Determining the Relationship between Gene Expression and Metabolite Concentration Changes. PLoS Comput Biol. 2014;10:e1003572.

39. Redestig H, Costa IG. Detection and interpretation of metabolite-transcript coresponses using combined profiling data. Bioinformatics. 2011;27:i357-65.

40. Patil KR, Nielsen J. Uncovering transcriptional regulation of metabolism by using metabolic network topology. Proc Natl Acad Sci U S A. 2005;102:2685-9.

41. Heiden MGV, Locasale JW, Swanson KD, Sharfi H, Heffron GJ, AmadorNoguez D, Christofk HR, Wagner G, Rabinowitz JD, Asara JM, Cantley LC. Evidence for an alternative glycolytic pathway in rapidly proliferating cells. Science (New York, NY). 2010;329:1492-9.

42. Davidson Shawn M, Vander Heiden Matthew G. METabolic Adaptations in the Tumor MYCroenvironment. Cell Metab. 2012;15:131-33. 